\title{
Luffer Track Optimization to Flexible System of Double Link Jib on Port Crane
}

\author{
Qingqing Yang, Yixiao Qin ", Chengzhi Fan, Shanshan Zhou \\ Mechanical Engineering Institution, Taiyuan University of Science and Technology, Taiyuan, China
}

Email address:

1438394060@qq.com (Qingqing Yang), qyx819@163.com (Yixiao Qin)

${ }^{*}$ Corresponding author

\section{To cite this article:}

Qingqing Yang, Yixiao Qin, Chengzhi Fan, Shanshan Zhou. Luffer Track Optimization to Flexible System of Double Link Jib on Port Crane. Science Research. Vol. 4, No. 5, 2016, pp. 107-111. doi: 10.11648/j.sr.20160405.11

Received: August 26, 2016; Accepted: September 8, 2016; Published: September 22, 2016

\begin{abstract}
Port crane is frequently used to load and unload cargoes in large mixed ship and hoisting equipment for ship manufacturing. Operation stability of the whole machine is largely determined by pros and cons of system performance of double link jib. Its optimization design has been the research focus in the industry, in the past, double link jib system is considered as a rigid link system to be designed, which ignores deflection change caused by its flexible deformation and excess energy consumption this may cause, however, double link jib system will be considered flexible deformation system in the paper, parameter optimization design is done for its main structure sizes, realizing the optimization of each lever's size in the jib system, cargoes could move approximately along the path of the horizontal direction when with payloads during luffing motion, so that luffing driving mechanism works with more energy-efficient and more smoothly. This study can make the construction of four-link mechanism more reasonable, more safe and reliable for the whole machine, which is a design method of green optimization in loading and unloading machine field.
\end{abstract}

Keywords : Port Crane, Luffer Track, Double Link Jib, Optimization of Flexible System

\section{Introduction}

Port crane is a growing steadily demand in port loading and unloading with rapid development of international logistics industry and shipbuilding Industry, at the same time, loading and unloading efficiently and hoisting precisely on equipment put forward to higher requirements to port crane working performance, therefore the optimization design of double link jib system of port crane has been a hot research topic in industry for a long time. This paper conducts a comprehensive study on the optimization design for luffing mechanism of portal slewing crane, mathematical model is established for luffing mechanism optimization design of portal slewing crane, with an aim of making crampon hinge end point to move horizontally, and of satisfying the value of maximum and minimum amplitude, and of saving material, etc. And the optimization result is satisfactory [1]. The main girders of 450 $\mathrm{t}$ bais-rail port crane was taken as the optimized object, the weight of main girders was set as the objective function; genetic algorithm was applied for optimization design, and the optimal dimensions of the main section were finally obtained by using finite element software [2]. The paper describes an implementation of genetic search methods in the optimal design of structural systems with a mix of continuous, integer and discrete design variables [3]. A portal crane was analyzed as rigid body model through this program. The simulation results were compared with the design criterion of arm system. A kind of new method for port crane's arm system design and optimization was offered [4]. Based on the structure analysis method of space motion mechanism, this work proposed a rapid modeling method without computer aided engineering preprocessor for the motion-changing process of four-bar linkage mechanism of combinatorial jib in portal crane. The study focused on the finite element model of four-bar linkage mechanism of combinatorial jib [5]. In order to realize the rapid visual modeling luffing mechanism of portal crane, a special visual modeling platform based on OpenGL and $\mathrm{C}$ mixed programming in the Windows XP operation system for four-bar linkage structure was developed in this paper [6]. Intelligent analysis and knowledge reuse for combinatorial jib 
of portal crane are realized, an expert system for finite element analysis was developed based on knowledge engineering [7]. It is deduced by combining the interpolated reproducing kernel particle method with the boundary integral equation method which aims to solve elastic mechanics plane stress [8]. As a core component of four-bar linkage portal crane, the design of combination boom will directly affect the performance of the machine, a detailed element model was established and each mode of vibration was calculated in this paper [9]. A brand-new jib-bridge luffing system of crane was brought out based on analysis of the limitation to handle containers of the portal crane. The system is provided with novel structure and advantages, the design methods are expounded of equalizing horizontal and balancing deadweight for the jib-bridge system. The optimizing design for the equalizing system ensured cargoes to move horizontally in luffing process, the design method of balancing system achieved ideally effect [10]. The arm frame system is the central part in the design of a portal crane. The purpose of this program was to assure that the load moves approximately along the path of the horizontal direction in the process of hoisting, through the optimization of the bars' length in the arm frame system [11]. In the past, the four-bar jib has been regarded as rigid link system to study, a new class of pseudo-global optimization procedures for solving formidable optimization problems were present [12]. The deflection phenomenon will be generated in head of arm frame system, because of the elastic deformation of the metal rod, it is found that the portal frame response dominates elastic behavior [13]. In the past, double link jib regarded as rigid link system has always been to study. But in fact, when crane with capacity and being under loading, deflecting appears in the head of jib system because of the flexible deformation of the metal bars, making the track of cargoes changing in horizontal direction, based on optimization design of rigid four-bar linkage, generally flexible deformation will increase fluctuation of cargoes in horizontal direction with amplitude variation. This will cause excessive energy consumption of luffing mechanism, which is also not conducive to the smooth working of the luffing mechanism, even affects the whole safety. Crane with horizontal luffing system is often used in gantry crane: that is, displacement path with loads is a horizontal line or similar to the horizontal line in the luffing course, which saves driving power of luffing mechanism, so the specific design is needed for the corresponding amplitude system in order that the cargoes path is along the horizontal line or track movement is close to a horizontal line in the luffing course, goods lift compensation device is used. Jib system is proposed as double link jib system of flexible deformation in this paper, and the optimization of the flexible jib system is based on rigid optimization, based on the basic theory of finite element and structural mechanics knowledge, static deformation analysis and dynamic characteristics analysis are studied to flexible jib system, the optimization objective function of the flexible jib system is re-established, and the optimization of each lever's size has been done. Thus making portal crane with payloads driven more stably in luffing motion, meanwhile luffing mechanism is more energy efficiency and environmental protection.

\section{Luffer Track Optimization to Flexible System of Double Link Jib}

Base on optimal design in rigid systems of four-bar linkage of port crane, the flexible deflection of the boom head with different amplitude is analyzed, and the flexible deformation curve is fitted, the optimization of flexible double link jib system is proposed through quadratic optimization in this study, so that cargoes could move approximately along the path of the horizontal direction when with payloads in luffing motion.

First, jib system is regarded as rigid four-link, and the main optimization of the jib system is to optimize the sizes of each pole.

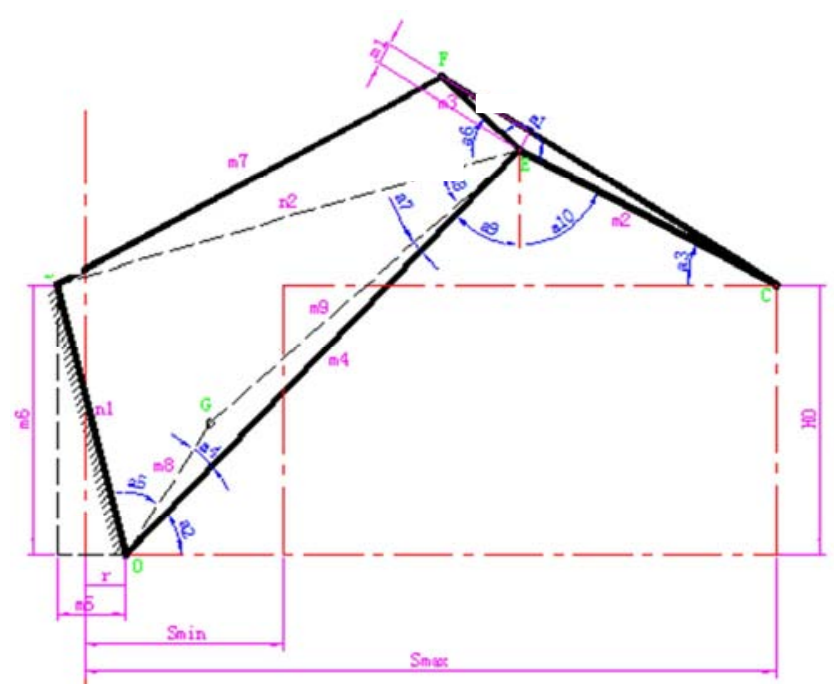

Fig. 1. Schematic diagram of the jib system.

As shown in Fig.1, there are seven design variables for the optimization of each lever's size of double link jib system.

$$
\begin{aligned}
\boldsymbol{x} & =\left[x_{1}, x_{2}, x_{3}, x_{4}, x_{5}, x_{6}, x_{7}\right]^{\mathrm{T}} \\
& =\left[m_{1}, m_{2}, m_{3}, m_{4}, m_{5}, m_{6}, m_{7}\right]^{\mathrm{T}}
\end{aligned}
$$

Due to the optimization problem of double link jib system, involving some port crane design parameter, such as the maximum amplitude $S_{\max }$ and minimum amplitude $S_{\min }$, lifting height $H_{0}$ etc. Therefore, it can be used as a known quantity, and the rest each lever's size in the arm frame system are viewed as the unknown quantity.

Beam moving along the path of the horizontal direction in luffing process is treated as optimization objective, the object function is established on size optimization of port crane jib system, first task was to make sure motion trajectory of beam head $\mathrm{C}$.

The motion trajectory and the objective function of $\mathrm{C}$ points are established by interrelationships of optimizing variables in 
luffing process, when the system component is set to a rigid body, being obtained from geometric relations between the four-link rods of double link jib.

$$
\begin{aligned}
& S_{\max }-m_{4} \cos \alpha_{2 \min }=m_{2} \cos \alpha_{3 \min } \\
& m_{4} \sin \alpha_{2 \min }-H_{0}=m_{2} \sin \alpha_{3 \min }
\end{aligned}
$$

The main jib swinging angle at the maximum amplitude position was obtained by the equation calculated

$$
\alpha_{2 \min }=\arcsin \left(\frac{d_{1} d_{3}+l_{d}}{d_{3}{ }^{2}+d_{2}{ }^{2}}\right)
$$

Here,

$$
l_{d}=\sqrt{d_{1}{ }^{2} d_{3}{ }^{2}-\left(d_{3}{ }^{2}+d_{2}{ }^{2}\right)\left(d_{1}{ }^{2}-d_{2}{ }^{2}\right)}
$$

The swinging angle of main $\mathrm{jib}$ at the a little amplitude position

$$
\alpha_{2 \max }=\arcsin \left(\frac{d_{4} d_{3}+m_{d}}{d_{3}{ }^{2}+d_{5}{ }^{2}}\right)
$$

Here,

$$
m_{d}=\sqrt{d_{4}{ }^{2} d_{3}{ }^{2}-\left(d_{3}{ }^{2}+d_{5}{ }^{2}\right)\left(d_{4}{ }^{2}-d_{5}{ }^{2}\right)}
$$

In the swing angle of the main jib in luffing process $\alpha_{2 \min } \leq \alpha_{2} \leq \alpha_{2 \max }$, the swing angle is divided into $\mathrm{N}$ parts, calculating $\mathrm{N}+1$ position, in any one of positions, swing angle of main arm

$$
\alpha_{2 i}=\alpha_{2 \min }+(i-1) \Delta \alpha_{2} \quad(i=1,2, \cdots, \mathrm{N}+1)
$$

Here, $\Delta \alpha_{2}=\frac{\alpha_{2 \max }-\alpha_{2 \min }}{\mathrm{N}}$

Vertical displacement of beam of $\mathrm{N}+1$ jib systems

$$
y_{C i}=m_{4} \sin \alpha_{2 i} i=1,2, \cdots, \mathrm{N}+1
$$

And taking minimizing of accumulative value of displacement variation as objective functions

$$
\min F(\boldsymbol{x})=\min \sum_{i} \mid y_{C(i+1)}-y_{C i}
$$

To meet the requirements of different constraints in double link jib system, in addition to design requirements for structures, double link jib system should take the correlation between design variables into consideration.

Whether the correlation between design variables is established well or badly affects directly the accuracy of the optimization results, therefore the correlation between design variables was an essential prerequisite to produce optimal solution.

Positions of each swing angle of main jib in luffing process, the high point $y_{C \max }$ and low point $y_{C \text { min }}$ of luffing trajectory of beam head, calculating height difference of luffing trajectory.

$$
y_{C \max }-y_{C \min } \leq 0.03\left(S_{\max }-S_{\min }\right)
$$

That is, limiting amplitude for cargoes in the horizontal direction by optimization design of double link jib is constraint conditions, which is got.

$$
g(\boldsymbol{x})=y_{C \max }-y_{C \min }-0.03\left(S_{\max }-S_{\min }\right) \leq 0
$$

After sizes of each bar are devised by the preliminary optimization of rigid double link jib above, and this scheme is used as the initial value of flexible optimization, the variation of flexible displacement in beam head of jib system takes the vertical displacement of the head into account to construct the objective function of the flexible jib system.

According to variable amplitude of the jib system under full loads condition, the purpose of moving approximately along the path of the horizontal direction is realized. In this paper, the design variables and constraints of jib system are not changed, but the objective function is reset.

By adopting the method of finite element static analysis of truss structure, $N+1$ flexible deflection of head of beam in the vertical direction in jib system is obtained, finding the minimum value of the vertical displacement change as the objective function, the objective function for the optimization of the flexible arm is

$$
F(x)=\min \sum_{i}\left|y_{C i}+\Delta_{i}-H_{0}\right|
$$

Where, $C$ point coordinate value in $\mathrm{y}$ direction after optimization of the rigid body is $y_{C i}, \Delta_{i}$ is flexible deformation for finite element analysis of body structure when jib system is used as flexible body.

Taking $25 \mathrm{t}$ portal crane as an example, technical parameters are known the maximum amplitude $S_{\max }=33000 \mathrm{~mm}$ and the minimum amplitude $S_{\min }=9500 \mathrm{~mm}$, lifting height above ground $\mathrm{H}_{0}=13175 \mathrm{~mm}$, when double link $\mathrm{jib}$ is designed as the rigid system, the value $\mathrm{H}_{0}=13175 \mathrm{~mm}$, which can meet the needs of the lifting height; When double link jib is designed as the actual flexible system, the value $H_{0}=13175+\Delta$, and $\Delta \geq 0$ through many optimization, that is because the lowest point of the flexible double link jib should be bigger than $13175 \mathrm{~mm}$ when considering to lift cargoes of rated lifting weight. Optimization of the main jib angle is divided into 100 equal parts, getting 101 range position, swing angles of the main jib at each position $\alpha_{2 i}=\alpha_{2 \min }+(i-1) \Delta \alpha_{2} i=1,2, \cdots \cdots 101$.

Seven bar dimensions of jib system are taken as the design variables, objective functions such as the formula (8) and (11) adopting continuous variable hybrid optimization method. The optimized results of jib system obtained are shown in table 1. Seven variables in the optimization design of jib system is shown in the first line, the data provided is the initial value of the optimization shown in the second line, the results of flexible deformation rated loads endured by rigid optimization is shown in the third line, the results based on flexible optimization is shown in the fourth line, the results of flexible deformation of rated loads endured by flexible 
optimization is shown in the fifth line.

Table 1. Optimization data of jib system $/ \mathrm{mm}$.

\begin{tabular}{lllllllll}
\hline \multicolumn{1}{c}{ Design variables } & \multirow{2}{*}{ ( } \\
\cline { 2 - 8 } & $\boldsymbol{x}_{\mathbf{1}}$ & $\boldsymbol{x}_{\mathbf{2}}$ & $\boldsymbol{x}_{\mathbf{3}}$ & $\boldsymbol{x}_{\mathbf{4}}$ & $\boldsymbol{x}_{\mathbf{5}}$ & $\boldsymbol{x}_{\mathbf{6}}$ & $\boldsymbol{x}_{\mathbf{7}}$ & \\
\hline (b) & 530 & 13200 & 4200 & 26500 & 3660 & 10500 & 21900 & 650 \\
(c) & 770 & 13336 & 4293 & 26456 & 3572 & 10505 & 22030 & 430 \\
(d) & 610 & 14567 & 4079 & 27413 & 3507 & 10452 & 22886 & 160 \\
(c) & 610 & 14567 & 4079 & 27413 & 3507 & 10452 & 22886 & 150 \\
\hline
\end{tabular}

In table 1, @Fluctuation value of boom head in horizontal direction, (b) Initial design, (c) Flexible deformation rated loads endured by rigid optimization, (d) Flexible optimization. (e) Flexible deformation rated loads endured by flexible optimization.

Among them, there are 74 times of iteration in the process of optimization, the minimum objective function of the flexible jib system is $336 \mathrm{~mm}$, because variable amplitude movement is carried out in the case of full loads. The optimization is based on the rigid optimization, from a perspective of flexible deformation, considering the luffing jib system produced a considerable amount of displacement, therefore, the method of optimization has certain practical significance in contact with the actual project.

In order to reflect more accurately the optimization effect of jib system, through three states mentioned above: initial value, flexible deformation and flexible optimization. the track of head of beam are respectively fitted by data, forming different deflection trajectories of head vertical direction in luffing process of port crane jib system.

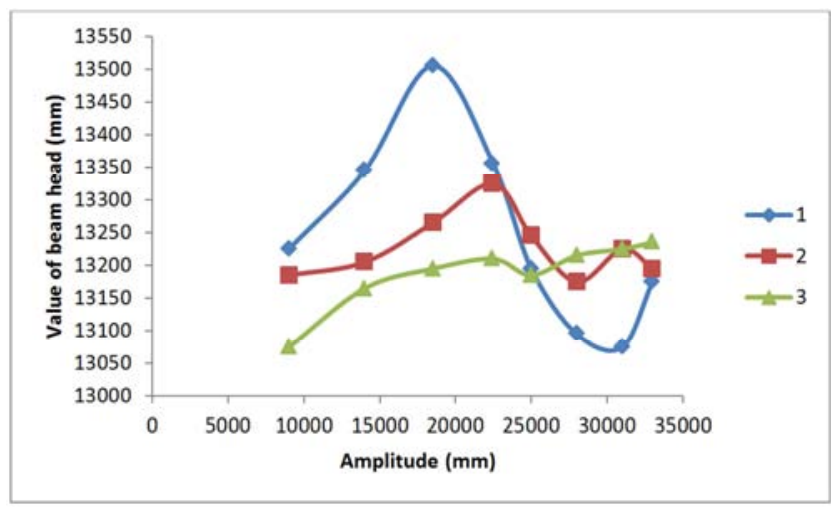

Fig. 2. Comparison chart of various tracks for head of beam by optimization.

Labels of diagram above are as follows: 1. Flexible deformation rated loads endured by rigid optimization. 2 . Flexible deformation of rated loads endured by flexible optimization. 3. Flexible optimization with hook being no-loads

The track of head of beam at three states is shown in Fig. 2 when jib system moves under variable amplitude. From the Fig.2, being clearly seen: the maximum height difference of the initial value is about $650 \mathrm{~mm}$, when variable amplitude was conducted in flexible boom system subjecting to full loads, drawing its running track after flexible deformation, the optimization of the flexible jib system is optimized again based on the rigid optimization, and from the maximum amplitude range to the minimum amplitude, the maximum height difference is about $160 \mathrm{~mm}$, and running trajectory of head of beam is similar to the decreasing curve, which just achieved complement each other with tracks of head of beam after flexible deformation, this is similar to the effect of precision design basic research in this paper. The horizontal wave value of the optimization results based on the rigid rod under the normal loads is $430 \mathrm{~mm}$, which could be down to $150 \mathrm{~mm}$ after based on flexible optimization, finally, the purpose of going along the path of the horizontal direction for cargoes is achieved with the flexible jib system of port crane being under rated loads in the normal loading and unloading operation.

\section{Conclusions}

1) Based on the rigid optimization for double link jib system of the port crane, the influence of the flexible deflection on the double link jib is considered again. In order to make the port crane achieve the purpose of moving more close to the path of the horizontal direction with full loads and amplitude movement in jib system, secondary energy-efficient, green design based on flexible deformation was achieved aimed at double link jib sizes. Thus making routine operation of port crane be more stable in more energy saving and environmental protection.

2) The method is an innovation design method of double link jib system on port crane. In this paper, be showed by the study that flexible optimization aimed to port crane jib system is of practical significance, therefore the optimization method has practical application value for port crane precision manufacturing.

\section{Acknowledgements}

This research was supported by the Natural Science Foundation of Shanxi Province of China (Grant No. 2013011022-6), the UIT of Shanxi Province of China (Grant No. 2014277) and the National Natural Science Foundation of China (Grant No. 51478290)

\section{References}

[1] Luo Z H. Optimization Design for Luffing Mechanism of Portal Slewing Crane [J]. Applied Mechanics \& Materials, 2014, 577:305-309.

[2] Wang M, Yu-Jie L I. Optimization Design for Main Girders of Gantry Crane Based on Genetic Algorithm [J]. Road Machinery \& Mechanization, 2011.

[3] C. Y. LIN, P. HAJELA. Genetic algorithms in optimization problems with discrete and integer design variables. Engineering Optimization, 1992, 19 (4): 309-327

[4] B Xiao, J. P. Guo, Research of the Amplitude Movement of Four-bar Linkage Arm by Multi bod Analysis. Journal of Wu Han University of Technology (2010). 
[5] Tong S G, Jun-Xu G E, Yan-Ling W U, et al. Rapid modeling method for combinatorial jib of portal crane [J]. Journal of Zhejiang University, 2009, 43 (11):2085-2090.

[6] Tong S G, Zhao Q. OpenGL based visual modeling for luffing mechanism of portal crane [C]. International Conference on Information Science and Engineering. IEEE, 2010:4617-4620.

[7] Zhao Q, Tong S G. Knowledge-based FEA system for combinatorial jib of portal crane [C]. Information Science and Engineering (ICISE), 2010 2nd International Conference on. IEEE, 2010:5147-5150.

[8] Qin Y X, Xie W T, Ren H P, et al. Crane hook stress analysis upon boundary interpolated reproducing kernel particle method [J]. Engineering Analysis with Boundary Elements, 2016, 63:74-81.

[9] Xu L J. Simulation Analysis about Dynamic Characteristics of Four-Bar Linkage Portal Crane's Jib Based on the Lifting Loads
[C], Sixth International Conference on Intelligent Systems Design and Engineering Applications. IEEE Computer Society, 2015:719-722.

[10] Yong-Zhi L I, Chen D F. New luffing system of portal crane and its design method [J]. Journal of Engineering Design, 2006, 13(4):232-235.

[11] Zheng X L, Li M D, Zheng H R, et al. Optimization and Simulation of Portable Crane's Arm System Based on MATLAB [J]. Advanced Materials Research, 2012, 479-481: 1955-1958.

[12] Sherali H D, Ganesan V. A Pseudo-Global Optimization Approach with Application to the Design of Containerships [J]. Journal of Global Optimization, 2003, 26 (4):335-360.

[13] Jacobs L D, Kosbab B D, Leon R T, et al. Seismic Behavior of a Jumbo Container Crane Including Uplift [J]. Earthquake Spectra, 2011, 27 (3):745-773. 